\title{
AN ADAPTIVE OSPF ROUTING STRATEGY \\ - BASED ON BANDWIDTH ESTIMATION - AND A SIMULATION PLATFORM FOR NETWORK PERFORMANCE ANALYSIS
}

\author{
Tatiana Brito Pereira and Lee Luan Ling
}

\begin{abstract}
Routing strategies play an essential role in network traffic distribution and consequently influence network performance. Adaptive routing requires that decisions on network condition be made so that network resources can be better used. An adaptive mechanism also generates additional updating messages over the network, inevitably augmenting the amount of traffic circulating therein. This paper has the goal of presenting an adaplive OSPF routing strategy based on bandwidth estimation. The estimation of effective bandwidths carried out as a function of traffic load and desired quality assists each router in its decision making. This paper also describes a simulation platform having a general structure for testing and analyzing any adaptive routing strategy.
\end{abstract}

Keywords: Adaplive routing, effective bandwidth estimation. OSPF, network performance parameters.

Resumo - Estratégias de roteamento exercem papel fundamental na distribuição do tráfego pela rede, conseqüentemente influenciando o desempenho da rede. $\mathrm{O}$ roteamento adaptativo toma suas decisões baseando-se nas condições da rede, de modo a melhor utilizar-se de seus recursos. Mecanismos adaptativos também geram mensagens adicionais de atualização na rede, aumentando de forma inevitável a quantidade de tráfego circulante. Este trabalho tem como objetivo apresentar uma estratégia de roteamento OSPF adaptativa baseada em estimações de banda. Estimações da banda efetiva (funçào da carga de tráfego e da qualidade desejada para o enlace) auxiliam cada roteador em suas tomadas de decisão. O trabalho descreve também uma plataforma de simulação para teste e análise de estratégias de roteamento adaptativas.

Palavras-chave: Roteamento adaptativo, estimação da banda efetiva, OSPF, parâmetros de desempenho de rede.

\section{INTRODUCTION}

The main function of a routing protocol can be summarized as the following: the determination and the learning of network topology and the selection of the best

Tatiana Brito Pereira and Lee Luan Ling are with the Laboratory of Pattem Recognition and Communication Networks (LRPRC). Communication Department (DECOM). School of Electrical and Computing Engineering (FEEC) of State University of Campinas (UNICAMP). Campinas - SP. Brazil. 13083-970. Phone: -5519 37883811, Fax: $\quad-55 \quad 19 \quad 32891395 . \quad$ E-mails: tatibpleefa decom.fee.unicamp.br. This project was partially supported by CAPES - Brazil and Ericsson Research do Brasil. Tatiana Brito Pereira currently is with CPqD Foundation. Campinas - SP. Brazil. 13086-902. Phone -55 19 37057252. Email: tpereira $a$ cpgd.com.br routing path. The OSPF protocol (Open Shortest Path First - RFC 2328) is an efficient and widely used link-state routing prolocol, having its routing decision making based on some pre-defined but usually static link costs.

Using the traditional OSPF protocol, packets are supposed to be routed through the least cost path, also called "the shortest path". The determination of the shortest path is based on static link costs, thereby changing path only when network components become unreachable or new network components are added. Network traffic congestion doesn't change the previously determined routing path, thus hardly are packets routed into other paths even when many of them are able to offer better end-to-end performance.

Measurements from the Internet indicate that there exist alternative paths capable of offering higher bandwidths and lower round-trip delay for $80 \%$ of selected traffic paths [19]. A major reason of this fact can include poor interdomain routing policies and/or inadequate intra-domain routing protocols.

Aiming at higher link utilization, adaptive routing has been widely investigated [3] [4] [5] [6] [7] [8] [11]. The major concept of adaptive routing is seeking a more efficient routing process based on current network condition by associating a lower link or path cost with those less used link resources. increasing interests in the utilization of less explored links. It is expected that better network performance in terms of packet throughput and delay can be achieved [1] [2].

However, adaptive routing mechanisms can also generate additional updating messages, increasing the amount of traffic circulating in networks, buffer occupancy, packet transfer delay, as well as packet drop rate. No doubt does a badly designed routing strategy and ill-controlling mechanism degrade or compromise network performance. This includes the so-called best path oscillation, resulting from the adaptation mechanism that chooses among multibest routing alternatives, however unnecessarily increasing the link utilization rate, overloading the network and possibly making the network unstable.

Many techniques have been proposed to deal with the problem of path oscillations in order to achieve stable routing [3] [4] [5] [6] [7]. Section 5 presents a new method for routing information updating and path oscillation control in the context of the proposed adaptive routing strategy.

The adaptive routing mechanism periodically monitors the situation/condition of the network environment. Metrics such as average link throughput together with mean buffer utilization [2] [8] [9], minimum packet transfer delay path [10] [11] and link utilization percentage [3] [7] are used. Although these measurements can accurately demonstrate 
the current network condition. each one presents advantages and disadvantages.

An example of this sort is the detemination of bandwidths for a given traffic load in the adaptive routing strategy. Under this context of qualitatively defined bandwidth, we recommend the use of effective bandwidth. which is the amount of bandwidth necessary to a traffic flow so that the required quality of end-to-end network service can be maintained

An intelligent adaptive routing mechanism should be not only capable of adjusting the routing efficiency according to network load. but also facilitating the management of network traffic load and link quality by the network administrator; including link bandwidth estimations in the adaptive routing strategy. Effective bandwidth to be adopted in this work is altematively defined as an amount of link bandwidth necessary to maintain certain required network link traffic quality which is specified by either maximum packet dropped rate or maximum transfer delay, or both of them. In Section 3, we detail our effective bandwidth approach being incorporated into the adaptive OSPF routing strategy proposed in this work

This work aims at investigating the behavior of the proposed adaptive OSPF routing strategy, which relies on bandwidth estimates to characterize link condition. to specify desired link qualities and to choose the best outgoing link for input traffic routing.

An additional important contribution is a simulation platform for network performance parameter analysis as well as new routing protocol testing. More specifically, we contributed with an adaptive router node model under the OPNET network simulator environment suitable for routing strategy analysis and validation.

\section{THE OSPF ROUTING PROTOCOL}

The OSPF routing protocol is a link state interior routing protocol. By "interior routing protocol", we mean that the OSPF is a protocol employed in the context of a collection of networks and routers that exchange routing information inrough a common routing protocol [12]. A collection of networks and routers operating under the same routing protocol forms a so-called Autonomous System (AS). In link state protocols, each router of the Autonomous System maintains a database (called as a link state database) containing information to specify the AS topology [12].

The AS topology can be determined by running the Hello protocol. Periodical exchanges of Hello packets enable the establishment and maintenance of neighbor relations between two AS routers. An inmediate benefit is the quick detection of any link failure, allowing quick repercussions around the entire network. Specifically, we do not need to deal with link failure detection due to the Hello Protocol having already been found implemented and taken a part of the OSPF protocol.

Adjacent relations are established from the router neighbor list. In principle adjacent relations have nothing to do with physical short distance and not all pairs of neighbor routers become adjacent routers. Instead, the rules that establish an adjacency relation strongly depend on the network topology also on the network technology. No further discussion about adjacent relations will be given here. How ever. it is worth mentioning that neighbor routers become adjacent in order to exchange routing information and only adjacent routers exchange information of link state database description.

An adjacent graph of an AS describes the routing protocol packet flows and. more specifically, the LSU (Link State Update) messages throughoul the AS. An LSU inessage reports link state database updating. Each LSU brings a set of LSAs (Limk State Achertisements).

The state of an interface (or a router link), which is detemined by the router identification unit via a RouterLSA, provides the following information: the link type, the network mask or IP interface address, the neighbor router ID and the respective router interface/link cost.

All participating routers of an AS hold the same link state database. The local status of a particular router is registered in this database. i.e. its interfaces and reachable neighbors. Each Router reports its local status in the AS by flooding messages so that the local link state database can be constantly updated. From this database, each router searches for the shortest path (called route) to all other routers using the Dijkstra algorithm [13]. All routers simultaneously run the same algorithm. each one building its routing tree by setting itself as a tree root. Once having the shortest path tree built, the routing table is prompt for the use.

The OSPF mechanism allows the update of the link state database and consequently the updating of routing tables in cases of network topological changes. However, these mechanisms lack the assessment of network traffic conditions, e.g.e traffic congestion. Thus, any link congestion in the network does not affect or change the OSPF routing.

\section{THE CONIPUTATION OF EFFECTIVE BANDWIDTHS}

Although there is no universally accepted definition for effective bandwidth. conceptually it is well established. That is, in this work the effective bandwidth is viewed as the minimum link capacity capable of assuring the required link quality for the given traffic. Commonly required quality are packet loss rate and packet transfer delay (or transfer delay variation, known as jitter). In fact the estimation of effective bandwidth becomes gradually important and popular, especially in network dimensioning and design. with the evolution of network technology that currently focuses on: high-speed links, multimedia applications and QoS required connections, etc.

In this work the effective bandwidth estimation is used as a way to not only measure network link conditions, but also to explicitly provide measurements for link quality in terms of packet loss rate. The link condition or link occupation is specified through traffic characterization in terms of the following parameters: PPR (Peak Packet Rate), SPR (Sustainable Packet Rate, or simply "mean rate") and MBS (Maximum Burst Size). Interesting enough is that these traffic characterization parameters (PPR, SPR, MBS) are related to the desired PLR (Packet Loss Rate), normally set 
by the network designer. through an effective bandwidth estimation algorithm.

More precisely we propose the use of effective bandwidth estimates in link cost computation so that cost estimates are able to reflect link loads in routing decision processes according to a desirable link quality vision. By this "desirable link quality vision" we mean that the traffic load will be viewed according to the desired PLR.

Most of existing bandwidth estimation methods are based either on an admissible loss probability (e.g. [14]) or the worst packet delay (e.g.. [15]) as a form of desired quality specification. In this work, we concentrate our attention on the first approach. that is, we adapt a generalized version of the loss based bandwidth estimation method proposed in [14]. Sections 3.1 and 3.2 are dedicated to the description of the original effective bandwidth estimation method proposed by Kesidis. et al [14] and the generalization proposed [16] is adopted in this work.

\subsection{KESIDIS' METHOD FOR EFFECTIVE BANDWIDTH ESTIMATION}

The effective bandwidth estimation method proposed in [14] based on the Large Deviation Principle requires that an admissible loss probability obey the following criterion:

where

$$
P\{X>b\} \leq \exp (-b \times \delta) \quad \text { (Equation 1) }
$$

$P\{X>b\} \quad$ is the admissible loss probability,

$X \quad$ is a random variable representing the

$b \quad$ is the buffer size (in bytes),

$\delta \quad$ is a scaling constant.

From Equation (1), the effective bandwidth estimates can be obtained for various kinds of traffic sources. Specifically Equations (2) to (7) summarize the procedure of obtaining the effective bandwidth $c$ for a Markovian ONOFF Fluid traffic source [16]:

$$
\begin{aligned}
& c=\alpha+\sqrt{\alpha^{2}+\beta} \\
& \alpha=\frac{1}{2 \delta}\left(P P R \times \delta-\frac{1}{T_{o n}}-\frac{1}{T_{\text {off }}}\right) \\
& \beta=\frac{P P R}{\delta \times T_{\text {off }}} \\
& T_{n n}=\frac{M B S}{P P R} \\
& T_{o f f}=M B S\left(\frac{1}{S P R}-\frac{1}{P P R}\right)
\end{aligned}
$$

$$
\exp (-b \times \delta)=P L R
$$

(Equation 7)

Equation (7) introduces the desired link quality in terms of the packet loss rate PLR, which in its turn is the function of the buffer size $b$ and a scale constant $\delta$. The traffic characterization parameters (PPR, SPR e MBS) appearing in Equations (3) to (6) describe some intermediate calculation values: $T_{m}, T_{o f f} \alpha$ and $\beta$. Finally the effective bandwidth $c$ is computed directly from Equation (2) as a function of $\alpha$ and $\beta$.

\subsection{THE ADOPTED METHOD FOR EFFECTIVE BANDWIDTH ESTIMATION}

The method of effective bandwidth estimation adopted in this work was originally proposed in [16], which can be viewed as a generalization of that proposed by Kesidis, et al [14] by incorporating the Hurst self-similar parameter $(H)$. This generalization is necessary due to the fact that when traffic is self-similar, Kesidis' method does not always provide a good approximation, in fact underestimating the effective bandwidth for a range of buffer sizes [16]. As a result, under that assumption of the Markovian On/Off Fluid source model, Equations (1) and (7), respectively, become

$$
P\{X>b\} \leq \exp \left(-\delta \times b^{2(1-H)}\right)
$$

(Equation 8)

and

$$
\exp \left(-\delta \times b^{2(1-H)}\right)=P L R
$$

(Equation 9)

Notice that the Hurst parameter $(H)$ is an important traffic characterization parameter capable of measuring the degree of traffic's self-similarity and long range dependence. The estimation of parameter $\mathrm{H}$ can be done via either the variance method or by the wavelet approach, which is the most accurate one

An optimization factor $f_{0}$ that helps to circumvent a limitation of the Large Deviation Principle (non-moderate buffer sizes) in effective bandwidth estimation by this generalized version in order to obtain more realistic estimates for different buffer sizes was recommended in [16] as the following.

$$
f_{0}=\frac{1}{2} \operatorname{arcsen} h(H \times \ln b)
$$

(Equation 10)

This optimization factor $f_{0}$ was empirically derived as a function of the buffer size $b$ and of the Hurst parameter $H$. Thus, the optimized effective bandwidth $\left(c_{0}\right)$ is obtained as

$$
c_{0}=f_{0}^{-1} \times c
$$

(Equation 11) 


\section{THE PROPOSED ADAPTIVE OSPF ROUTING STRATEGY}

An adaptive routing algorithm has two proceedings: the link cost estimations and the "policy for link cost updating decision". In the first proceeding. the costs to be used in the next shortest/best path update period is computed based on the previous traffic load information while in the second proceeding, the costs are analyzed so that the decision on cost updating can be made.

The proposed adaptive routing algorithm was developed under the OPNET Modeler network simulator en ironment. The updates of link costs based on effective bandwidth estimates are carried out for each active router interface. Figure 1 shows events involved in routing and the flow of information among the related active router interfaces, the bandwidth estimation modules and the traditional OSPF module. Arrows denote the occurrence of events and data reading.

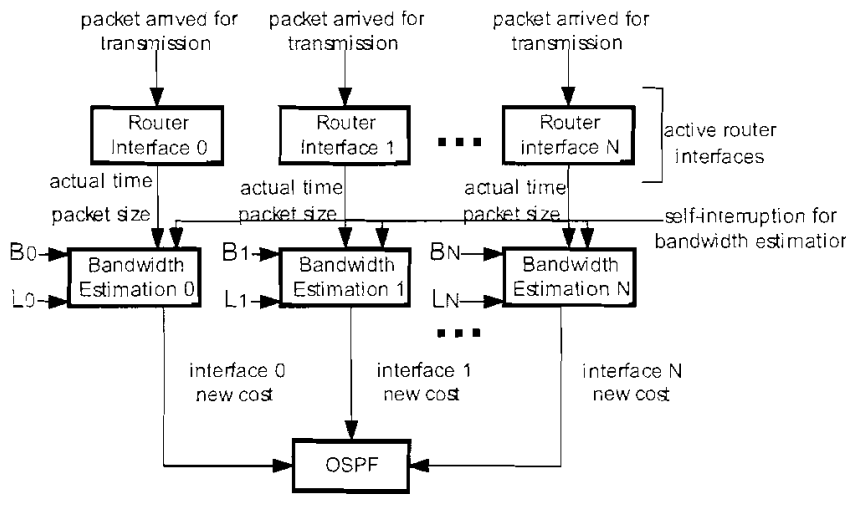

$\mathrm{Bx}=$ buffer size of Router Interface $\mathrm{x}$

$L x=$ wished loss rate for the link associated to the Router Interface $x$

Figure I -- The Adaptive OSPF Routing

Based on Bandwidth Estimation.

For each active router interface, there is a corresponding bandwidth estimation module. The correspondence between an estimation module and a router interface is done through an interface number $\{0,1, \ldots, N\}$. Each time a packet arrives at the input of a router interface. information such as irrival time and packet size is provided to the corresponding bandwidth estimation module. Each bandwidth estimation module keeps saving these two pieces of information (arrival time and packet size). Bandwidth estimation is periodically performed from the scheduled self-interruptions. Each bandwidth estimation module computes the bandwidth demanded by the traffic iransmitted through the corresponding interface for the time period $[t-B E P, \mathrm{t}]$, based on the information such as the buffer size, the required link loss rate and the stored traffic trace, where $t$ is the current time and BEP is called the Bandwidth Estimation Period, the duration on which traffic trace is colleted and used for bandwidth estimation.

As a function of the current bandwidth estimate, the link cost corresponding to the considered router interface is given by:

$$
C_{l, t}=\left[10 \times\left(\frac{B E_{(t-B E P . t]}}{C T_{l}}\right)\right] \quad \text { (Equation 12) }
$$

where
$C_{1.1}$

$t$

$B E P$
$B E_{(t-B E P . t}$

$C T_{1}$

\lfloor\rfloor is the cost corresponding to the link $l$ at the time instant $t$. is the current time (time when occurring the effective bandwidth estimation). is the Bandwidth Estimation Period.

is the estimated bandwidth on the period $[t-B E P, \mathrm{t}]$.

is the total transmission capacity of the link $?$.

is the integer part of the real number $X$.
Notice that the new link cost is calculated immediately after effective bandwidth estimation and the cost update is done periodically every BEP time period. The new link cost is then passed to the OSPF module, together with the corresponding number of the intertace iñuvañ a NEW_COST_MESSAGE typed message. New link cost notification messages were named MEIF_COST MESSAGE. These messages flow between the bandwidth estimation modules (comesponding to each router interfacel and the OSPF module.

Since effective bandwidth estimation and then new link cost computation in each router interface every BEP period are performed independently among router interfaces, the OSPF module receives independent NEW_COST_MESSAGE messages from their bandwidth estimation modules every BEP period.

Upon receiving new costs, the OSPF module activates the procedure of "policy for link cost updating decision" to decide whether updating the costs and then flooding the network with this information or simply ignoring the incoming information. It is desirable that an update policy offers a good tradeoff between maximum utilization of current network traffic information and minimum updating overloads.

The state of a router interfaces is propagated through a special type of LSA named Router-LSA. A Router-LSA carries cost information of all active router interfaces. If the cost modification of at least one router interface justifies the generation of an updating message, all router interfaces will have their cost updated. This updating is done through the link state database interface costs updating and through the propagation of LSUs into the network. Finally, after receiving these LSUs, all other nodes will update their link state database, which is periodically used for the routing table re-building.

\section{THE POLICY FOR LINK COST UPDATING DECISION}

A major point of the proposed adaptive routing strategy, called "policy for link cost updating decision" is a sequence of tests to decide when the link cost updating at router interfaces and the propagation of this information occur.

Intuitively. it is easy to perceive that adaptive routing in principle can intelligently distribute traffic loads through 
the network by choosing better routes according to network conditions, increasing network throughput. Better throughput results can be achieved even if no updating control mechanism is used [17].

Besides the throughput issue, network delay and packet dropped rate are other two important issues. The introduction of routing updating messages (overloads) into networks can increase traffic intensity and cause larger packet transfer delay in buffers due to longer waiting time, and also may lead to an increasing in packet drop rates. Thus, the main issue in "policy for link cost updating decision" consists in the deternination of updating frequency (or updating periods) and the choice of the best updating action. Definitely this is a non-trivial task due to the difficulty in establishing the best tradeoff among high throughput, small delay and a low packet drop rate.

The adaptive routing algorithm proposed by Aceves [4] imposes that the updated link costs can be only propagated through links if they belong to one of the best paths so that traffic overloads can be easily controlled.

In an attempt to reduce routing oscillation and, therefore, link cost updating. Shaikh [7] [3] recommends the use of the so-called minimum cost criterion defined by the percentage of link utilization. This criterion assumes the same cost value for any link having its link utilization inferior to that pre-defined minimum value. For overload reduction the method adopted in [7] establishes a minimum time period between two consecutive updates, an upper bound for updating periods and random variation in updating periods for different links.

Apostolopoulos [5] establishes a threshold to trigger link cost updating action in order to reduce routing oscillation and uses a regressive clock to limit the updating rate.

Shaikh [6] tries to reduce overloads due to propagation of link state update information, and consequently route oscillations, through differential treatments for short and long flows. The link state is characterized only by those allocated resource flows with long duration and less bursty like. This artificial mechanism reduces considerably the updating propagation frequency and the updating overload.

The "policy for link cost updating decision" implemented in this work consists of the following controling mechanisms: a minimum cost. a maximum limit for link cost variation and a minimum limit for link cost updating. The justification of this strategy is given next.

When the link utilization is low and oscilates between zero and a mininum link utilization value, it is neither interesting to reflect this oscillation on link costs nor interesting to overload the network with cost update messages. The use of the minimum cost criterion. similar to that in [7] and [3], in which any utilization below this minimum threshold is upper aligned and the minimum cost will be applied. can avoid cost oscillation and reduce overloads.

The criterion of maximum limit for link cost variation, as induced by the proper name, controls the cost variation by limiting the maximum allowable link cost variation. avoiding situations where a destination route alternates intensely between two best paths.

The criterion of a minimum limit to the link cost variation has the goal of reducing the cost updating frequency. If the link cost variation is very low (for instance. oscilating between zero and a minimum limit), the unecessary generation and propagation of link cost messages should be avoided because the best paths probably change little.

Notice that the procedure of "policy for link cost updating decision" is necesary in order to impose limits to the link cost oscillation levels, to minimize link cost updating frequency and to reduce routing table rebuilding overloads.

\section{ADAPTIVE OSPF SCENARIO EXAMIPLE}

The simulation environment for the evaluation of the proposed adaptive OSPF routing strategy is shown in Figure 2 through an example network.

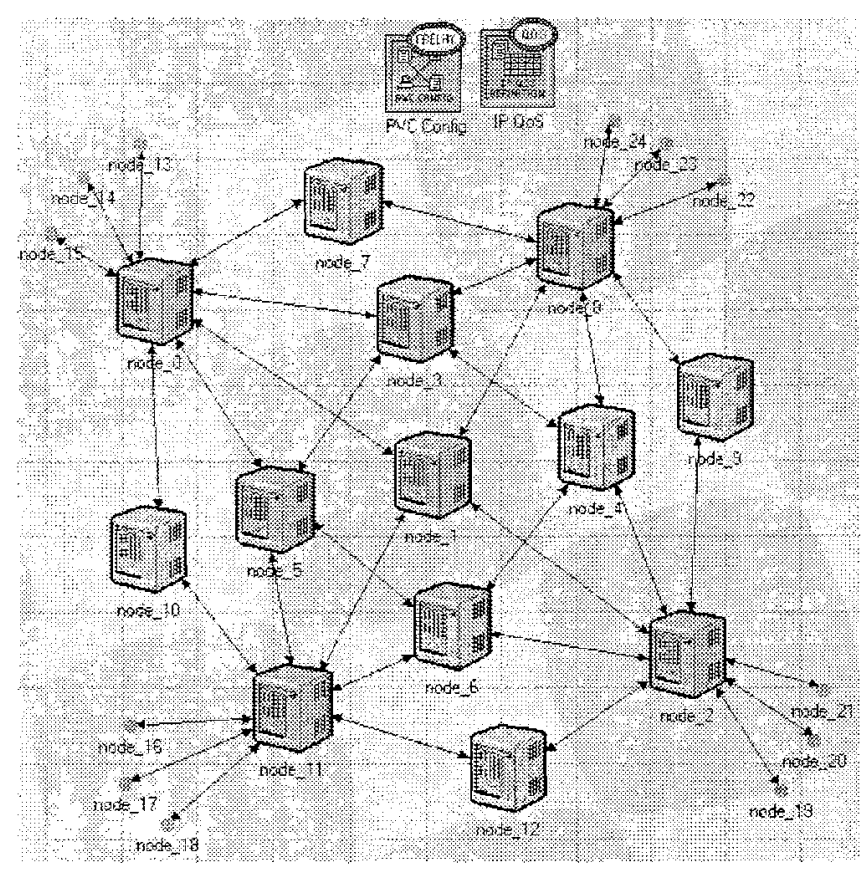

Figure 2 - Example Network for Simulation.

Each link in the example network operates under Frame Relay 4.0 Mbps and each node uses FIFO (First-In-FirstOut) stacks with a 70 Kbytes buffer in each output interface. The traffic introduced into the network is specified by the following information: <origin, destination $>$ pairs, IP packet generation start time and stop time, average time between packets (referred to as "average delta time") and average packet sizes.

Five traffic specifications (namely S1, S2, S3, S4 and S5) were selected for tests. For each specification, the traffic flow goes from the nodes 13-18 to the nodes 19-24 according to the following <origin, destination> pairs: $\langle 13,19\rangle,\langle 14,20\rangle,\langle 15.21\rangle,\langle 16,22\rangle,\langle 17,23\rangle,\langle 18,24\rangle$. For the performance comparison purposes, among these five traffic specification we maintain the common values for traffic parameters including the start and the stop times and the average packet sizes, and gradually increase the traffic intensity, therefore with the decreasing average delta time value in the following sequence order: S1, S2, S3, S4. $\mathrm{S} 5$. Table 1 lists the adopted traffic parameter values for the traffic specification S1 for each one of these source- 
destination pairs. In addition we assume that the delta time. known as interarrival time. for each source-destination pair is an independent and exponential variable, and the packet

\begin{tabular}{|l|l|l|l|l|}
\hline $\begin{array}{l}<\text { source, } \\
\text { destination }>\end{array}$ & $\begin{array}{l}\text { Start } \\
\text { Time }\end{array}$ & $\begin{array}{l}\text { Stop } \\
\text { Time }\end{array}$ & $\begin{array}{l}\text { Average } \\
\text { Delta } \\
\text { Time (us) }\end{array}$ & $\begin{array}{l}\text { Average } \\
\text { Size } \\
\text { (Bytes) }\end{array}$ \\
\hline$<13.19>$ & 200 & 1500 & 2800 & 600 \\
\hline$<14.20>$ & 700 & 2300 & 900 & 200 \\
\hline$<15.21>$ & 2000 & 3600 & 1500 & 350 \\
\hline$<16.22>$ & 400 & 1200 & 1700 & 300 \\
\hline$<17,23>$ & 800 & 1000 & 2600 & 450 \\
\hline$<18.24>$ & 1400 & 3600 & 1800 & 600 \\
\hline
\end{tabular}

Table 1. Traffic 1 Specification (Scenario S1)

\begin{tabular}{|l|l|l|l|l|l|}
\hline $\begin{array}{l}\text { <source, } \\
\text { destination }>\end{array}$ & \multicolumn{5}{|l}{ Average Delta Time (us) } \\
\cline { 2 - 6 } & S1 & S2 & S3 & S4 & S5 \\
\hline$<13.19>$ & 2800 & 2600 & 2500 & 2400 & 2300 \\
\hline$<14,20>$ & 900 & 800 & 700 & 650 & 500 \\
\hline$<15,21>$ & 1500 & 1300 & 1100 & 1000 & 950 \\
\hline$<16.22>$ & 1700 & 1600 & 1500 & 1300 & 1200 \\
\hline$<17,23>$ & 2600 & 2400 & 2300 & 2200 & 2000 \\
\hline$<18.24>$ & 1800 & 1700 & 1650 & 1600 & 1400 \\
\hline
\end{tabular}

Table 2. Average Delta Times - Scenarios S1 to S5.

sizes follow Poisson distributions. Table 2 shows the adopted average delta time values for each of the five traffic specifications.

Both the proposed adaptive and the traditional OSPF routing strategies [12] were simulated and tested under the same network environment and traffic specifications. In Section 7 we compare the simulation results obtained from analyzing these 10 different simulation scenarios (i.e., 5 traffic specification cases for each one of two routing strategies.)

Notice that the objective of studying different traffic specifications is to see how each adaptive routing strategy reacts to different network traffic loads and the degree of improvement and impacts that the adaptive routing strategy is able to offer with respect to the traditional static OSPF routing strategy.

\section{SIMULATION RESULTS}

This section presents the experimental investigation results for the above described 10 simulation scenarios. namely NET1 1a, NET1 1c, NET1 2a, NET1 2c.
NETl 3a. NETl 3c. NETl ta. NETl tc. NET1 5a and XET1_5c. The last two letters after a dash in the notation represent the traftic specification number (or type) and the routing strategy type ( "a " for the adaptive routing while "c" for the traditional or "conventional" one).

Figure 3 shows how the arerage hroughput evolves in terms of simulation time duration. Clearly the adaptive strategy outperforms the traditional OSPF one by providing higher network throughput for all tested lraffic specifications.

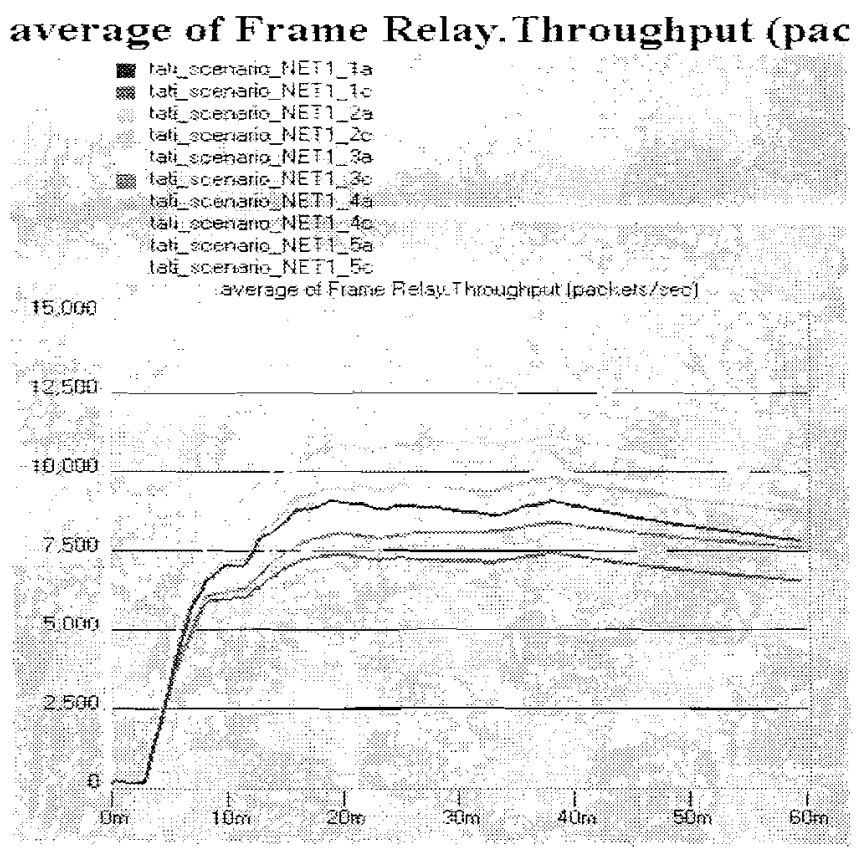

Figure 3 - Average Throughput X Time - Scenarios 1 to 5.

Figure 4 shows how network transfer delay varies in function of source traffic intensity and the influence of routing strategies. For scenario $\mathrm{Sl}$, with low traffic intensity the proposed adaptive routing mechanism offers only slightly smaller average delay than its traditional counterpart. However, when the traffic intensity becomes higher, as in the cases of Scenarios S2. S3, S4 and S5. the difference in measured average delay between two routing strategy types becomes evident that confirms the superiority of the adaptive routing means. Table 3 shows numerically the delay performance of these five traffic scenarios and clearly demonstrated the superiority of the adaptive strategy to the traditional OSPF. For packet dropped rate analysis, according to Figure 5 the adaptive strategy offers higher packet dropped rates in scenarios $\mathrm{S} 1$ and $\mathrm{S} 2$ and lower dropped rates in the traffic scenarios S3, S4 and S5 than the traditional routing means. 
average of Frame Relay.Delay (sec)

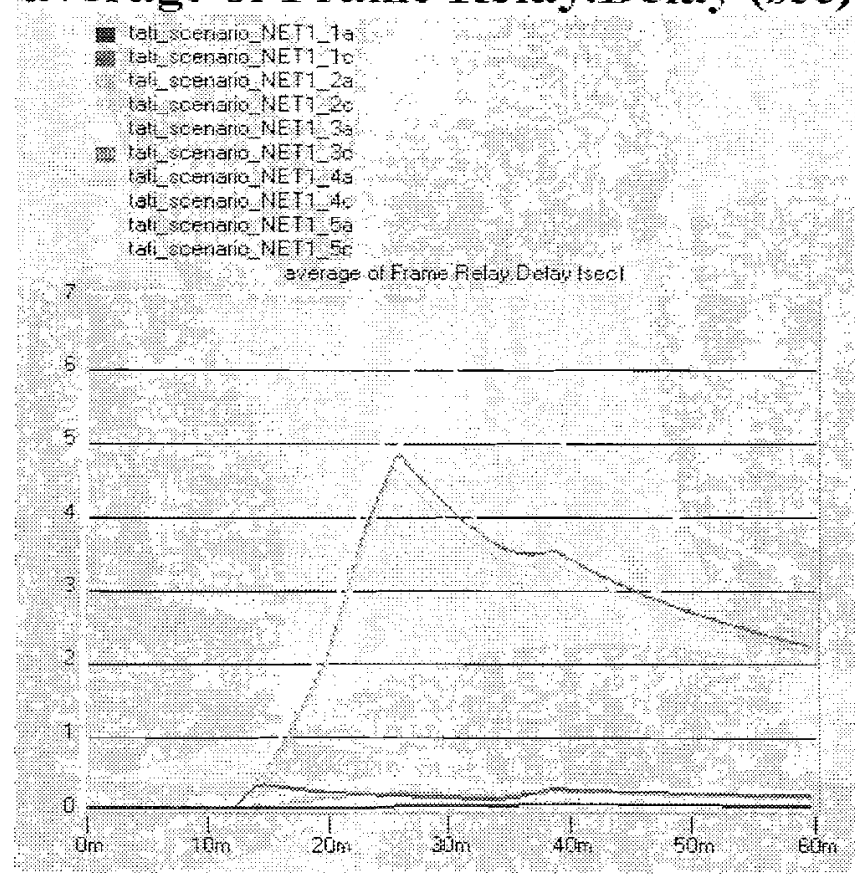

Figure 4 - Average Delay X Time - Scenarios 1 to 5.

\begin{tabular}{|c|c|c|}
\hline & Average Delay & \\
\hline $\begin{array}{l}\text { Traffic } \\
\text { Scenario }\end{array}$ & $\begin{array}{l}\text { Conventional } \\
\text { Strategy }\end{array}$ & $\begin{array}{l}\text { Adaptive } \\
\text { Strategy }\end{array}$ \\
\hline 1 & 0.165754 & 0.040759 \\
\hline 2 & 2.107570 & 0.146827 \\
\hline 3 & 2.208358 & 0.115827 \\
\hline 4 & 2.291846 & 0.132146 \\
\hline 5 & 3.179885 & 0.219044 \\
\hline
\end{tabular}

Table 3. Average Delay Results - Scenarios S1 to S5.

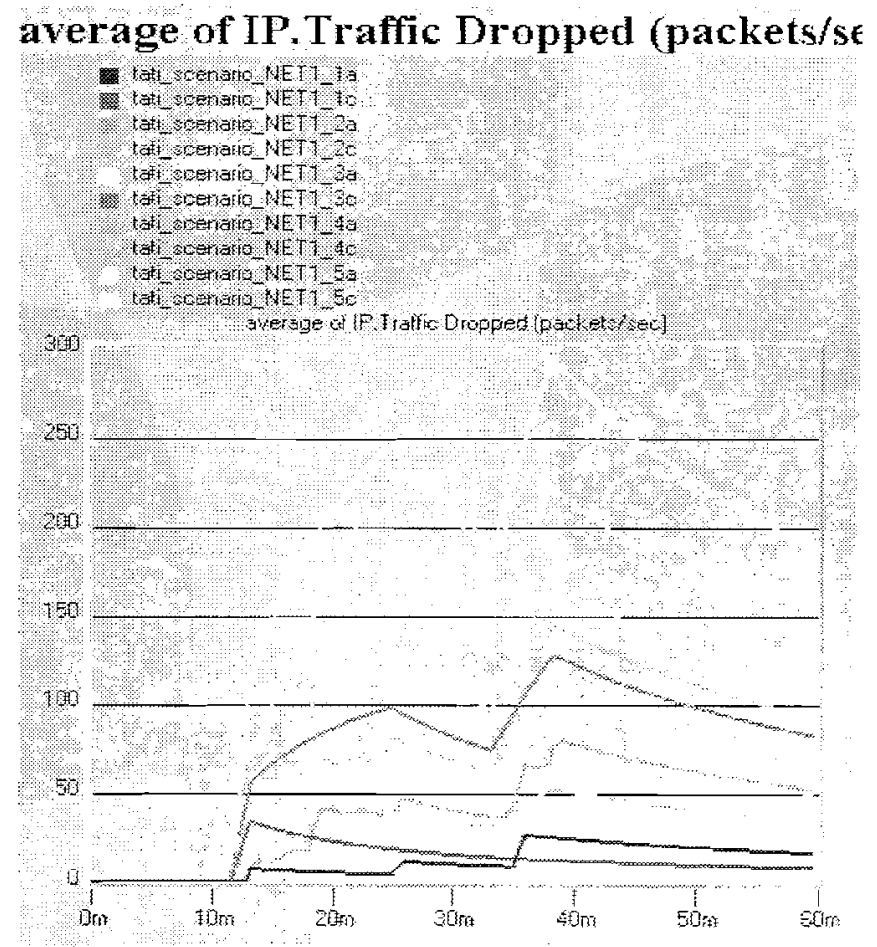

Figure 5 - Average Traffic Dropped Rate X Time Scenarios S1 to S5.
Based on these simulation results, the advantage of the adaptive routing strategy becomes evident, especially when the network traffic is intense.

\section{CONCLUSION}

In this paper, we proposed a link state based adaptive routing strategy with a novel route decision criterion that takes into account the concept of network link quality. The introduction of link quality concept in network and routing strategy analysis has the goal of providing quantitatively the quality of a link in terms of bandwidth needed for the incoming traffic flow and possibly offering a good alternative outgoing link for traffic routing. On purpose, we adapted the concept of the effective bandwidth to define the link quality that would offer some imaginary required service quality and therefore link a cost for the best routing decision capable of preserving this service quality.

In order to validate the proposed approach, network quality was evaluated experimentally by simulation in terms of average network throughput, packet transfer delay and packet drop rate. For the comparison purpose, the traditional OSPF routing was taken as a reference.

We have observed that the proposed adaptive routing strategy offers better route decisions than the traditional OSPF, which is intuitively expected due to the fact that adaptive routing can better distribute traffic loads among network links. An immediate consequence of this fact is higher network throughput that becomes even accentuated for high traffic intensity.

At the very first glance, one may conclude that the main drawback of an adaptive routing strategy is larger packet transfer delay due to the additional load, known as updating link cost messages, introduced into the network. Surprisingly enough we found that this may be the case when network traffic load is light, for example, Traffic Specification S1. However, for heavy traffic loads, as the cases of Traffic Specifications S2, S3, S4 and S5, the adaptive routing surpasses largely the traditional OSPF with lower packet transfer delay. In other words, the additional delay introduced by the updating messages has little effect in comparison with the incurred benefit offered by the alternative routes. Finally in terms of packet drop rates the use of an adaptive routing strategy becomes even more crucial whenever the network is dominated by high rate traffic flows. That is, alternative paths can avoid traffic congestion and therefore the discarding of packets.

Another contribution of this work is the simulation platform built under OPNET Modeler environment. Its modular characteristics make it a facility for testing any kind of routing strategies.

\section{REFERENCES}

[1] A. Khanna. J. Zinky. "The Revised ARPANET Routing Metric". 1989 ACM SIGCOMM, p. 45-56. 1989.

[a] D. W. Glazer. C. Tropper. "A New Metric for Dynamic Routing Algorithms". IEEE Transactions on Communications, Vol. 38. No. 3. Warch 1990.

[3] A. Shaikh. J. Rexford. K. G. Shin. "Dynamics of Quality-ofService Routing with Inaccurate Link-State Information", Lniversity of Michigan Technical Report. November 1997. 
[4] J. J. G. L. Acerss. M. Spohn. "Scalable Link-State lntemet Routing". Proceedings Sixth International Conference on Network Protocols. pp. 52-61. 1998.

[5] G. Apostolopoulos. R. Guerin. S. Kamat. S. Tripathi. "Qualiry of service based routing: A performance perspective". ACXI SIGCOMM. September 1998.

[6] A. Shaikh, J. Rexford. K. G. Shin. "Load-Sensitive Routing of Long-Lived IP Flows". Conference on Applications. Technologies. Architectures. and Protocols for Computer Communication. p. 215-266. 1999.

7] A. Shaikh, J. Rexford. K. G. Shin. "Evaluating the Impact of Stale Link State on Quality-of-Service Routing". IEEE ACM Transactions on Networking. Vol. 9. No. 2. April 2001.

[8] H. T. Kaur, K. S. Vastola. "The Tunability of Network Routing Using Online Simulation“. SCS Symposium on Performance Evaluation of Computer and Telecommunication Systems (SPECTS 2000). Vancouver. BC. July 2000.

[9] T. Ye. D. Harrison, B. Mo. B. Sikdar. H. T. Kaur. S. Kalyanaraman, B. Szymansti. K. Vastola. "Traffic Management and Network Control Lising Collaborative Online Simulation". [EEE Intcrnational Conference on Communications (ICC 2001), Vol. 1, p. 204-209, 2001.

[10] J. M. McQuillan. I. Richer, E. C. Rosen. "The New Routing Algorithm for the ARPANET". IEEE Transactions oll Communications, Vol. 28. No. 5. May 1980.

[11] S. Vutukury. S.. J. J. G. L. Aceves, "A Simple Approximation to Minimum-Dclay Routing". Proceedings of the Conference on Applications. Technologies. Architectures and Protocols for Computer Communication, pp. 227-238. 1999

[12] J. Moy. "OSPF Version 2". RFC 2328. April 1998.

[13] D. E. Comer. "Interligação em Rede com TCP/IP - Princípios. Protocolos e Arquitetura", Volume I, Editora Campus. 1998.

[14] G. Kesidis, J. Walrand. C. Chang, "Effective Bandwidth for Multiclass Markov Fluids and Other ATM Sources". IEEE Trans. Networking. vol. 1, August 1993.

[15] J. Le Boudec, "Network Calculus Made Easy", Technical Report EPFL-DI, December 1996.

[16] F. R. Perlingeiro, "Estudo de Caracterização de Tráfego e Alocação de Banda para Redes ATM". Tese de Mestrado apresentada à Faculdade de Engenharia Elétrica (FEEC) da Universidade Estadual de Campinas (UNICAMP). Julho 1999.

[17] T. B. Pereira, L. L. Ling, "Nerwork Performance Analysis of an Adaptive OSPF Routing Strategy - Effective Bandwidth Estimation", ITS 2002 (Intemational Telecommunications Symposium 2002), Natal - RN - Brazil. September 2002.

[18] T. B. Pereira, L. L. Ling, "An OPNET Modeler Based Simulation Platform for Adaptive Routing Evaluation", Proceedings of OPNETWORK 2002, Washington D. C. USA. August 2002.

[19] S. Savage, A. Collins, E. Hoffman. J. Snell. T. Anderson. "The End-to-End Effects of Internet Path Selection". 1999 ACM SIGCOMM Conference, pp. 289-299. September 1999.

Tatiana Brito Pereira has received Bachelor degree in Computer Science from UNESP, São José do Rio Preto - SP. in 1999 Honored by reaching the best performance of the 1999 Graduation Class. She is actually finishing her Master in Electrical and Computing Engineering (FEEC) of State University of Campinas , UNICAMP) and working with CPqD Foundation since October 2002. She has been active with routing strategy studies. network simulations using OPNET Network Simulator. traffic characterization and bandwidth estimations. She has technical publication and presentation in international conferences like OPNETWORK 2002 - Washington D. C.. USA. August $2002-$ and ITS 2002 - Natal. RN, Brazil, September 2002.

Lee Luan Ling has received a dcgree in Electrical Engineering (Electronics and Telecommunications) from USP, São Carlos - SP
(1980): a Master's degree in Electrical Engineering from LXIC A.TP. Campinas-SP (1984): and a Doctor degree and PosDoctor degree in Philosophy both from the School of Electrical Engineering. Comell Lniversiry - LSA (1991). He is currently a Tizular Professor of L.ICA.iP with sereral publications at national and intemational levels and chapters in books. His research interests areas are pattern reeognition and communication networks 